\title{
Supporting the sharing of research
}

\author{
Nature Cell Biology supports the wide dissemination of scientific findings both prior to and following publication of \\ primary research manuscripts.
}

W hen Marshall McLuhan referred to the electronically interdependent world of the 1960s as a global village, he could not have foreseen how apt this description would be for the digital age, where global communication barriers have been shattered and connectivity and information sharing are near instantaneous. Research has advanced with the times, embracing a digital existence that has seen it flourish as a collaborative, interactive and interdisciplinary enterprise on a scale that was not feasible a few short decades ago. The landscape of science communication and publishing has also continued to evolve to address the need of researchers and the general public for more readily accessible and discoverable knowledge.

One manner of rapid dissemination of scientific findings is through preprints manuscripts that are posted on dedicated online servers by authors prior to peerreviewed publication. Ask any physicist about preprints and they will point you to arXiv, the hugely popular server that was originally founded by Paul Ginsparg in 1991 specifically for that community. Over its 26-year existence, arXiv has diversified to cover physical and quantitative science more broadly, with an impressive 1.34-million preprints posted as of December 2017. In contrast, uptake of the preprint concept in biological communities has been slower. As an example, the q-bio section of arXiv that was established in 2003 to serve quantitative biologists only numbered $\sim 12,000$ preprints as of December 2017. Similarly, the Nature family's own Nature Precedings, which was launched in 2007 as a preprint server for the life sciences, listed only $\sim 5,000$ manuscripts by the time it ceased accepting new submissions in 2012. However, attitudes have been changing in recent years, with a surge of interest in preprints among biologists including scientist-driven, preprintchampioning initiatives such as ASAPbio (Accelerating Science and Publication in biology). This has been punctuated by the launch of several servers in the life sciences domain (for example, bioRxiv, PeerJ Preprints, Preprints.org and OSF Preprints) and a concomitant increase in life science preprints (http://go.nature.com/2BM8YZd), largely owing to the rapidly growing popularity of bioRxiv, the server established in 2013 by the Cold Spring Harbor Laboratory.
Among the several positives of preprints is the immediate availability of research findings to the worldwide scientific community, which many contrast to the often lengthy peer-review and revision process that precedes publication in a scientific journal. Preprint posting also allows researchers to obtain feedback from a wider pool of experts and to improve their work prior to submission, and offers a time-stamped, citable record that some argue can establish priority of discovery. A counterargument is that staking primacy claims might encourage posting of weak, premature studies. Indeed, preprints are not peer reviewed and may therefore include more preliminary findings that could be modified after further scrutiny. However, a main purpose of preprints is to encourage scientific discussion. Thus, the strength of data versus claims of primacy would be something for the research community and relevant stakeholders to evaluate when considering preprints, be it as a basis for further research, or in hiring and funding decisions. Using communityaccepted servers that have strong systems of curation and versioning (for example, to avoid plagiarism and duplicate or outdated versions) will aid in maintaining a clear record of the original posting and subsequent progress of a particular study.

It should be noted that the long-standing position of Nature Cell Biology and other Nature journals on preprints has remained unchanged - we support preprint posting on community servers, without this jeopardizing consideration of a manuscript submitted to the journal (for policy details see http:// go.nature.com/2jNR01P). The non-peerreviewed, originally submitted version of a manuscript may be posted on a preprint server at any time. Preprints posted in recognized community servers may also be cited in the reference lists of our papers, although caution is always advisable. If main claims or methodology rely on citing preprints, editors and referees reserve the right to request that statements be qualified, for example, through validation of key methods and presentation of data in the manuscript under consideration by the journal.

Following formal acceptance of a manuscript for publication, we strongly encourage authors to self-archive the accepted version six months after publication of the journal's final 'version of record' of the manuscript (for details of the full policy see http://go.nature. $\mathrm{com} / 2 \mathrm{nMzBuX})$. At the time of formal acceptance, we also ask authors who have posted a preprint to update it with a publication reference, DOI and link to the journal-published version of the article. Our policy is in line with the open-access mandates of the majority of funders, and to enable authors to meet these requirements, we also offer an automated, free Manuscript Deposition Service (http:// go.nature.com/2A2IdiF). This service involves automatic deposition of the accepted manuscript version to be made public six months after publication of the paper, and is available to authors whose funders have agreements with PubMed Central and Europe PubMed Central, and who have opted in during the submission process.

Beyond pre-publication posting and self-archiving, one of our more recent and groundbreaking content-sharing initiatives is SharedIt, a function that was launched in October 2016 across the whole Springer Nature portfolio, including Nature Cell Biology and other Nature journals. Through SharedIt, authors and subscribers are able to obtain links to free-to-read versions of primary research papers published in our journals immediately after publication. SharedIt links can be shared for non-commercial, personal use through e-mail, or on online platforms such as websites, repositories and social media. In its first year, this initiative was warmly embraced by our authors and readers, who used SharedIt links to access Springer Nature content over 3.25 million times (http:// go.nature.com/2iHEtMx).

These policies and initiatives represent our strong commitment to ensure that research findings are widely accessible, discoverable and shareable to enhance the dissemination of knowledge, aid collaboration and advance scientific discovery. We will continue to evolve our policies and efforts to better meet the needs of the scientific community, and as always, we welcome the thoughts of our authors and readers at ncb@nature.com.

Published online: 21 December 2017 https://doi.org/10.1038/s41556-017-0025-8 